\title{
ON A CLASS OF EXPANSIONS IN OSCILLATING FUNCTIONS ${ }^{\text {i* }}$
}

\author{
Byy A. C. Dixon.
}

[Received December 25th, 1904.-Read January 12th, 1905.]

In memoirs published in the tirst two volumes of Liouville's Journal, Liouville and Sturm considered transcendents satisfying differential equations of a certain type, and gave a proof that an arbitrary function could be expanded in a series of multiples of such functions. Many of the expansions used in physics are of the type in question. The proof given is not now accepted (see Burkhardt's Report, p. 759), and the object of the present paper is to give a sound proof, at least for functions which are analytical throughout the proposed range of validity of the expansions: the proof is actually of more general application than this, but there is no attempt to examine whether any function expansible in a Fourier series can also be expanded in the more general form. Another limitation is that the range of validity of the expansions is not supposed to reach to any critical point of the differential equation satisfied by the general term.

On the other hand, the expansions have a greater degree of generality than those of Liouville on account of the presence of four arbitrary real constants (restricted by an inequality) in the place of his two $(h, H)$.

In the simple case of Fourier's series the oscillating series

$$
\Sigma(\cos n x \cos n t+\sin n x \sin n t)
$$

is multiplied by $F(t)$ and integrated with respect to $t$. 'The resulting series converges, and use is made of un expression for the sum to " terms to find the sum to infinity. The same idea is carried out here for the wider class of expansions treated. There is a fundamental oscillatingr series $\Sigma \Phi$, whose terms are symmetric functions of $x$ and $t$; an expression is found for the sum of $n$ terms of this series by means of

* For references on the subject see a report on "Entwickelungen nach oscillirende" Funotionen," by H. Burkhardt, in the Jahresbericht der Deutschen Mathematiker-Fereinigung, now being issued. 
a contour integral ; the oscillating series is multiplied by $F(t)$ and integrated, and thus turned into a convergent series whose sum is $F(x)$.

2. In the differential equation

$$
\frac{d^{2} y}{d x^{2}}+(X+\lambda) \cdot y=0
$$

let $\lambda$ be a parameter, $X$ a function of $x$ independent of $\lambda$. Suppose that, for real values of $x$ between 0 and 1 inclusive, $X$ has no singularity and that its values are real throughout that range.

Derivatives with respect to $x$ and $\lambda$ will occur; they will be indicated by dashes and dots respectively.

Let $\phi, \psi, \chi, \omega^{*}$ be functions satisfying (1) and distinguished by the following initial conditions :-

when $x=0, \quad \phi=0, \quad \phi^{\prime}=1, \quad \psi=1, \quad \psi^{\prime}=0$;

when $x=1, \quad \chi=0, \quad \chi^{\prime}=1, \quad \omega=1, \quad \omega^{\prime}=0$.

Then, by the known theory of differential equations of the second order, $\phi^{\prime} \psi-\phi \psi^{\prime}, \chi^{\prime} \omega-\chi \omega^{\prime}$ are constants, and, in fact, each of them is equal to 1 .

Also $\phi \chi^{\prime}-\phi^{\prime} \chi, \psi \chi^{\prime}-\psi^{\prime} \chi, \omega \phi^{\prime}-\omega^{\prime} \phi, \omega \psi^{\prime}-\omega^{\prime} \psi$ are constants, that is, depend on $\lambda$ only ; let their values be $e, g, h, k$. Then

$$
\begin{array}{ll}
e=\phi(1)=-\chi(0), & h=\phi^{\prime}(1)=\omega(0), \\
g=\psi(1)=\chi^{\prime}(0), & k=\psi^{\prime}(1)=-\omega^{\prime}(0),
\end{array}
$$

and

$$
g h-e k=1 \text {. }
$$

We also have

$$
\begin{array}{ll}
\chi=g \phi-c \psi, & \omega=-k \phi+h \psi, \\
\phi=h_{\chi}+e \omega, & \psi=k_{\chi}+g \omega .
\end{array}
$$

3. From the theory of differential equations $\dagger$ we know that $\phi, \psi, \chi, \omega$ are analytical functions of $\lambda$ as well as of $x$, and that therefore $e, g$, $h, k$ are analytical functions of $\lambda$. This is true for all finite values of $\lambda$. There is, of course, an essential singularity at infinity, as will appear more fully from results that are to follow.

* Written $\phi x, \psi x, \ldots$, or $\phi(x), \psi(x), \ldots$ or $\phi(x, \lambda), \ldots$ when necessary.

+ See especially Poincaré, Acta Mathematica, Vol. xIIr., pp. 15, 16. Other references are given in Forsyth's Theory of Differential Equations, Vol. r., chap. ii. 
4. 'The more general equation

$$
\frac{d^{2} y}{d x^{2}}+(X+\lambda U) y=0
$$

may be reduced to the form (1) by a change of variables, if $U$ is a holomorphic function of $x$ only and is constantly positive, not zero, between the limits 0, 1. The reduction is made by taking $U^{\frac{1}{3}} y$ as the new dependent variable and $\int_{0}^{x} U^{b} d x / \int_{0}^{1} U^{\frac{b}{b}} d x$ as the new independent.

5. Suppose $\lambda_{1}$ to be a new value of $\lambda$ and $\phi_{1}, \psi_{1}, \ldots, e_{1}, g_{1}, \ldots$ to be what $\phi, \psi, \ldots, e, g, \ldots$ become when $\lambda_{1}$ takes the place of $\lambda$. Then

$$
\begin{gathered}
\phi^{\prime \prime}+(X+\lambda) \phi=0, \quad \chi_{1}^{\prime \prime}+\left(X+\lambda_{1}\right) \chi_{1}=0 . \\
\phi^{\prime \prime} \chi_{1}-\phi \chi_{1}^{\prime \prime}=\left(\lambda_{1}-\lambda\right) \phi \chi_{1}, \quad \phi^{\prime} \chi_{1}-\phi \chi_{1}^{\prime}=\left(\lambda_{1}-\lambda\right) \int \phi \chi_{1} d x .
\end{gathered}
$$

Take the limits to be 0 and 1 ; thus

$$
\left(\lambda_{1}-\lambda\right) \int_{0}^{1} \phi \chi_{1} d x=-\phi(1)-\chi_{1}(0)=e_{1}-e
$$

Similarly

$$
\begin{aligned}
& \left(\lambda_{1}-\lambda\right) \int_{0}^{1} \phi \omega_{1} d x=\phi^{\prime}(1)-\omega_{1}(0)=h-h_{1}, \\
& \left(\lambda_{1}-\lambda\right) \int_{0}^{1} \psi \chi_{1} d x=-\psi(1)+\chi_{1}^{\prime}(0)=g_{1}-g, \\
& \left(\lambda_{1}-\lambda\right) \int_{0}^{1} \psi \omega_{1} d x=\psi^{\prime}(1)+\omega_{1}^{\prime}(0)=k-k_{1} .
\end{aligned}
$$

If in these we diminish $\lambda_{1}-\lambda$ without limit, we have

$$
\left.\begin{array}{ll}
e=\int_{0}^{1} \phi \chi d x, & \dot{g}=\int_{0}^{1} \psi \chi d x, \\
\dot{h}=-\int_{0}^{1} \phi \omega d x, & \dot{k}=-\int_{0}^{1} \psi \omega d x .
\end{array}\right\}
$$

These results could have been found by differentiating the equations $\phi^{\prime \prime}+(X+\lambda) \phi=0, \ldots$ with respect to $\lambda$. Thus

But

$$
\dot{\phi}^{\prime \prime}+(X+\lambda) \dot{\phi}=-\phi \text {. }
$$

so that

$$
\chi^{\prime \prime}+(X+\lambda) \chi=0 \text {, }
$$

$$
x \dot{\phi}^{\prime \prime}-\chi^{\prime \prime} \dot{\phi}=-\phi x \text {. }
$$


Integrating between 0 and 1 , we have

$$
\dot{e}=\int_{0}^{1} \phi X d x
$$

and so for the rest.

()ther useful results are

$$
\begin{aligned}
\left(\lambda_{1}-\lambda\right) \int_{0}^{1} \phi \phi_{1} d x & =e_{1} h-e h_{1} \\
\left(\lambda_{1}-\lambda\right) \int_{0}^{1} \phi \psi_{1} d x & =g_{1} h-e k_{1}-1 \\
\left(\lambda_{1}-\lambda\right) \int_{0}^{1} \phi \psi d x & =-g h_{1}+e_{1} k+1 \\
\left(\lambda_{1}-\lambda\right) \int_{0}^{1} \psi \psi \psi_{1} d x & =g_{1} k-g k_{1} \\
\left(\lambda_{1}-\lambda\right) \int_{0}^{1} \chi \chi_{1} d x & =g e_{1}-e g_{1} \\
\left(\lambda_{1}-\lambda\right) \int_{0}^{1} \omega \chi_{1} d x & =g_{1} h-e_{1} k-1 \\
\left(\lambda_{1}-\lambda\right) \int_{0}^{1} \chi \omega_{1} d x & =-g h_{1}+e k_{1}+1 \\
\left(\lambda_{1}-\lambda\right) \int_{0}^{1} \omega \omega_{1} d x & =k h_{1}-k_{1} h \\
\int_{0}^{1} \phi \psi &
\end{aligned}
$$

6. Now let $E, G, H, H, L$ be real constants, and suppose $\lambda, \lambda_{1}$ to be values satisfying the condition*

$$
E e+G g+H h+K k=2 L .
$$

We shall show that, if a certain condition of inequality is fultilled by $E, G, H, K, L$, the values of $\lambda$ satisfying (5) are all real.

- The reader will perhaps find it u help to take first one or two special cases, for instance, that in which $G, J, K, I$. vanish, and that in which $E=K=0, G=H=L=1$. It is hardly neressary to recommend conparison with the trigonometrical expansions which arise when $X=0$. 
For, by the fornulæ of $\$ 5$,

$$
\begin{aligned}
\left(\lambda_{1}-\lambda\right) & \int_{0}^{1}\left(E_{\chi_{1}}-H \omega_{1}+L \psi_{1}\right)\left(E E_{X}-H \omega+L \psi\right) d x \\
= & E^{2}\left(g e_{1}-e g_{1}\right)-E H\left(g_{1} h-e_{1} k-g h_{1}+e k_{1}\right) \\
& \quad+H^{2}\left(k h_{1}-k_{1} h\right)+L E\left(2 g_{1}-2 g\right)-L H\left(2 k-2 k_{1}\right)+L^{2}\left(g_{1} k-g k_{1}\right) \\
= & \left(E e_{1}+H h_{1}-2 L\right)(E g+H k)-(E e+H h-2 L)\left(E g_{1}+H k_{1}\right) \\
\quad & \quad+L^{2}\left(g_{1} k-g k_{1}\right) \\
= & -\left(G g_{1}+K k_{1}\right)(E g+H k)+(G g+K k)\left(E g_{1}+H k_{1}\right)+L^{2}\left(g_{1} k-g k_{1}\right) \\
= & \left(L^{2}+E K-G H\right)\left(g_{1} k-g k_{1}\right) .
\end{aligned}
$$

Hence, unless $\lambda_{1}=\lambda$.

$\int_{0}^{1}\left\{\left(E_{\chi_{1}}-H \omega_{1}+L \psi \psi_{1}\right)\left(E_{\chi}-H \omega+L \psi\right)+\left(G H-E K-L^{2}\right) \psi \psi_{1}\right\} d x=0$.

Suppose then that $\lambda$ is complex; the conjugate quantity will also satisfy the condition (5), and may be taken as $\lambda_{1}$. Thus $\chi, \chi_{1}$ and $\omega, \omega_{1}$ and $\psi, \psi_{1}$ are also conjugate, and, if

$$
G H>E K+L^{2},
$$

the equation (6) is impossible, the expression on the left having a positive value.

If $G H=E K+L^{2}$, (6) cannot now be true unless

$$
E_{\chi}-H \omega+L \psi=0
$$

that is,

$$
E+k L=0, \quad-H+g L=0 .
$$

Thus $g, k$ are real and are equal to $g_{1}, k_{1}$. But

$$
g_{1} k-g k_{1}=\left(\lambda_{1}-\lambda\right) \int_{0}^{1} \psi \psi_{1} d x
$$

a purely imaginary quantity, not zero. Hence $\lambda$ cannot be complex, even when $G H=E K+L^{2}$.

When $E, H, L$ all vanish, this argument fails, but the same result may be established by considering $\int_{11}^{1}\left(G \chi_{1}-K \omega_{1}-L \phi_{1}\right)(G \chi-K \omega-L \phi) d x$.

7. We shall then suppose $G H \nless E K+L^{2}$, so that the values of $\lambda$ satisfying the condition (5) must be real. They will generally be distinct also. If not, we should have

$$
E \dot{e}+G \dot{y}+H \dot{h}+K \dot{k}=0,
$$


that is, $\quad \int_{0}^{1}\{E \phi \chi+G \psi \chi-H \phi \omega-K \psi \omega\} d x=0$.

The subject of integration is here

or

$$
\begin{gathered}
(E \phi+G \psi)(g \phi-e \psi)+(H \phi+K \psi)(k \phi-h \psi) \\
\phi^{2}(E g+H k)+\phi \psi(G g-E e+K k-H h)-\psi^{2}(G e+K h),
\end{gathered}
$$

and is of constant sign if

$$
(G g-E e+K k-H h)^{2}<-4(E g+H k)(G e+K h),
$$

or if

$$
(G g+E e+K k+H h)^{2}<4(G g+K k)(E e+H h)-4(E g+H k)(G e+K h)
$$

or

$$
4(G H-E K)(g h-e k) \text {, }
$$

that is, if

$$
L^{2}<G H-E K \text {. }
$$

Hence, in this case, a double value of $\lambda$ cannot occur.

If $L^{2}=G H-E K$, a double value of $\lambda$ will occur only when the same subject of integration vanishes identically, that is, when

$$
E g+H k=0=G e+K h, \quad G g+K k=E e+H h=L .
$$

These conditions give

$$
L e=(G g+K k) e-(G e+K h) g=-K,
$$

and similarly $\quad L g=H, \quad L h=G, \quad L k=-E$.

Hence this case cannot occur when $L=0$. It does happen when $X$ is constant and

$$
E=K=0, \quad G=H= \pm L .
$$

A triple value of $\lambda$ cannot occur. This would mean the further condition

$$
E \ddot{e}+G \ddot{g}+H \ddot{h}+K \ddot{k}=0 \text {, }
$$

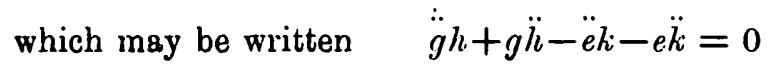

or

$$
\frac{\partial^{2}}{\partial \lambda^{2}}(g h-e k)=2(\dot{g} \dot{h}-\dot{e} \dot{k}) .
$$

Thus we should have $\ddot{g} \dot{h}-\dot{e} \dot{k}=0$, that is,

$$
\frac{1}{2} \int_{0}^{1} \int_{0}^{1}(\phi x \psi t-\phi t \psi x)\left(\chi^{x} \omega t-\chi t \omega x\right) d^{3} x d^{2} t=0,
$$

ou account of the formulæ (3).

But

$$
\chi x \omega t-\chi t \omega x=\phi x \psi t-\phi t \psi x,
$$


and therefore this double integral is positive and cannot vanish unless, for all values of $x$ and $t, \phi x / \psi x=\phi t / \psi t=a$ constant,

which is impossible, since $\phi^{\prime} \psi-\phi \varphi^{\prime}{ }^{\prime}=1$.

8. Let $x, t$ be two quantities, real, positive, and $<1$. Consider the value of $\int \Omega(x, t, \lambda) d \lambda$ where $\Omega(x, t, \lambda)$ stands for

$$
\frac{E \phi x \chi t+G \psi x \chi t-H \phi x \omega t-K \psi x \omega t+L(\phi x \psi t-\psi x \phi t)}{E e+G g+H h+K k-2 L}
$$

ind the integral is taken round a closerl curve in the $\lambda$ plane which does not pass through any point at which the denominator vanishes. The only singularities of the subject of integration for finite values of $\lambda$ are poles at the points where

$$
E e+G g+H h+K h=2 L .
$$

At any such point, supposed to be a simple zero of the denominator, the derivative of the denominator is

$$
\int_{0}^{1}(E \phi \chi+G \psi \chi-H \phi \omega-K \psi \omega) d x,
$$

and the numerator is a symmetric function of $x, t$, since it may be written

$$
\begin{aligned}
(E g+H k) \phi x \phi t+(-E e-H h+L) \phi x \psi t & +(G g+K k-L) \psi x \phi t \\
& -(G e+K h) \psi x \psi t .
\end{aligned}
$$

These expressions are respectively the denominator and numerator of the residue. In the exceptional case (see $\S 7$ ) when the denominator has a double zero, the numerator vanishes also, as may be seen by taking it in the form last written. Thus the subject of integration has only a simple pole, the residue being

$$
2 \frac{(E \dot{g}+H \dot{h}) \phi x \phi t+(G \dot{g}+K \dot{k})(\phi x \psi t+\psi x \phi t)-(G \dot{e}+K \dot{h}) \psi x \psi t}{E \ddot{e}+G \dot{g}+H \ddot{h}+K \dot{k}}
$$

But now

$$
\begin{aligned}
& E: G: H: K::-k: h: g:-e, \\
& \ddot{g} h+g \ddot{h}-\ddot{e} k-e \ddot{k}=2(e \dot{k}-\dot{g} \dot{h}) .
\end{aligned}
$$$$
\text { and }
$$

The residue is therefore

$$
\begin{aligned}
& \frac{(\dot{g} k-g \dot{k}) \phi x \phi t+(e \dot{k}-h \dot{g})(\phi x \psi t+\phi t \psi x)+(\dot{e} h-e \dot{h}) \psi x \psi t}{\dot{g} \dot{\bar{t}}-\dot{e} \dot{k}} \\
& \frac{\phi x \phi t \int_{0}^{1} \psi^{2} d x-(\phi x \psi t+\phi t \psi x) \int_{0}^{1} \phi \psi d x+\psi x \psi t \int_{0}^{1} \phi^{2} d x}{\frac{1}{2} \int_{0}^{1} \int_{0}^{1}(\phi x \psi t-\phi t \psi x)^{2} d x d t} .
\end{aligned}
$$


Hence

$$
\int \Omega(x, t, \lambda) d \lambda=2 \iota \pi \Sigma \Phi(x, t, \lambda)
$$

where $\Phi(x, t, \lambda)$ stands for

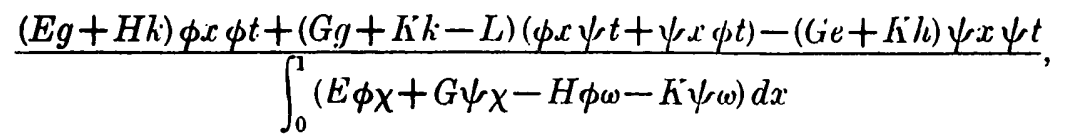

and the summation cover's all the values of $\lambda$, within the contour of integration, at which

$$
E e+G g+H h+K k=2 L \text {. }
$$

and, if two of these values of $\lambda$ coincide, the corresponding terms in the summation are to be replaced by the expression (7). If we suppose the values of $\lambda$ at which poles of $\Omega$ occur, in exsending order of numerical mugnitude, to be $\lambda_{1}, \lambda_{2}, \lambda_{3}, \ldots$, we thus have two expressions for the sum of $n$ terms of the series

$$
\Phi\left(x, t, \lambda_{1}\right)+\Phi\left(x, t, \lambda_{2}\right)+\ldots,
$$

namely, $(2 \iota \pi)^{-1} \int \Omega(x, t, \lambda) d \lambda$ and $(2 \iota \pi)^{-1} \int \Omega(t, x, \lambda) d \lambda$, the integrals being taken along some closed path, say the circle $|\lambda|=l$, which encloses. $\lambda_{1}, \lambda_{2}, \ldots, \lambda_{n}$, and not $\lambda_{n+1}, \lambda_{n+2}, \ldots$ In order to test the convergency of the series we may examine further these expressions for the sum to. $n$ terms. It will be found that the series does not converge as it stands.

9. We need to know the orders of magnitude of $\phi, \psi, \ldots$ when $\lambda$ is $a$ great complex quantity. Now when $X=0$ and $\lambda=(\alpha+\iota \beta)^{2}$ the most general solution of the equation (1) is

$$
y=A \cos \{(\alpha+\imath \beta) x+a+\iota b
$$

where $A, a, b$ are constants, $A$ being complex and $a, b$ real. Here then

$$
\begin{aligned}
|y|^{2} & =|A|^{2}\left\{\cosh ^{2}(\beta x+b)-\sin ^{2}(\alpha x+a)\right\} \\
& =|A|^{2}\left\{\sinh ^{2}(\beta x+b)+\cos ^{2}(\alpha x+a)\right\} .
\end{aligned}
$$

Thus $|y|$ oscillates between $|A| \cosh (\beta x+b)$ and $|A| \sinh (\beta x+b)$.

$$
\text { Again, } \quad \begin{aligned}
\left|y^{\prime}\right|^{2} & \left.=|\lambda||A|^{2} i \sinh ^{2}(\beta x+b)+\sin ^{2}(\alpha x+a)\right\} \\
& =|\lambda||A|^{2} ; \cosh ^{2}(\beta x+b)-\cos ^{2}(\alpha x+a)_{i}^{\prime},
\end{aligned}
$$

which oscillates in like manner.

$$
\text { But } \quad\left|\lambda y^{2}\right|+\left|y^{\prime}\right|^{2}=\left|\lambda A^{2}\right| \cosh (2 \beta x+2 b),
$$

which increases steadily with $x$. On the other hand, $\left|\lambda y^{2}\right|-\left|y^{\prime}\right|^{2}$ oscillates between $\pm\left|\lambda A^{2}\right|$, and in fact $\lambda y^{2}+y^{\prime 2}$ is constant and equal to $\lambda A^{2}$. 'These results are approximately true when $X$ is variable, as will now be shown. 
The following lemma will be used. If $v$ is defined by the differential equation

$$
u^{\prime \prime}=4 \mu^{2} u
$$

where $\mu$ is a function of $x$ that lies between two positive constants $\mu_{1}, \mu_{2}$ $\left(\mu_{1}>\mu_{2}\right)$ so long as $x$ is between 0 and 1 , and by the further conditions that when $x=0, w=1, w^{\prime}=0$, then $w$ is constantly between $\cosh 2 \mu_{1} x$ and $\cosh 2 \mu_{2} x$ so long as $x$ is between 0 and 1 .

For let

$$
W=\cosh 2 \mu_{1} x-w .
$$

Then

$$
W^{\prime \prime}=4\left(\mu_{1}^{2}-\mu^{2}\right) \cosh 2 \mu_{1} x+4 \mu^{2} W,
$$

and therefore $W^{\prime}$ increases with $x$ so long as $W \nless 0$, while $W$ increases with $x$ so long as $W^{\prime}>0$. Initially $W$ and $W^{\prime}$ both vanish and therefore both increase with $x$ and are always positive, that is, $\cosh 2 \mu_{1} x>w$. Similarly $w>\cosh 2 \mu_{2} x$.

10. Let $\lambda=\rho+\iota \sigma,|X+\lambda|=Q$, and put $y=u \exp \iota$, so that $\rho, \sigma$, $u, v$ are real, and $\rho=a^{2}-\beta^{2},|\lambda|=l=a^{2}+\beta^{2}$. Then

$$
y^{\prime \prime}=\left(u u^{\prime \prime}-u v^{\prime 2}+2 u u^{\prime} v^{\prime}+u u v^{\prime \prime}\right) e^{\iota "},
$$

and therefore $u=-u v^{2}+2 u u^{\prime} v^{\prime}+u v^{\prime \prime}+u(X+\rho+\imath \sigma)=0$.

Separating the real and inaginary parts, we have

$$
u^{\prime \prime}-u v^{\prime 2}+u(X+\rho)=0, \quad 2 u^{\prime} v^{\prime}+u v^{\prime \prime}+u \sigma=0 .
$$

Now put $\quad w=\left|(X+\lambda) y^{2}\right|+\left|y^{\prime}\right|^{2}=Q u^{3}+u^{\prime 2}+u^{2} v^{\prime 2}$.

Then $\quad v^{\prime}=Q^{\prime} u^{2}+2 u u^{\prime}\left(u^{\prime \prime}+Q u-u v^{\prime 2}\right)+2 u v^{\prime}\left(u v^{\prime \prime}+2 u u^{\prime} v^{\prime}\right)$

$$
=Q^{\prime} u^{2}+2 u u^{\prime}(Q-X-\rho)-2 \sigma u^{2} v^{\prime} ;
$$

$$
\begin{aligned}
w^{\prime \prime} & =Q^{\prime \prime} u^{2}+2 u u^{\prime}\left(2 Q^{\prime}-X^{\prime}\right)+2(Q-X-\rho)\left(u u^{\prime \prime}+u^{\prime 2}\right)-2 \sigma\left(u^{2} v^{\prime \prime}+2 u u^{\prime} v^{\prime}\right) \\
& =Q^{\prime \prime} u^{2}+2 u u^{\prime}\left(2 Q^{\prime}-X^{\prime}\right)+2(Q-X-\rho) u .
\end{aligned}
$$

after substitution for $u^{\prime \prime}, v^{\prime \prime}$ and reduction.

Now the terms $Q^{\prime \prime} u^{2}+2 u u^{\prime}\left(2 Q^{\prime}-X^{\prime}\right)$ ure generally insignificaut compared with the other's, for $Q^{\prime}=X^{\prime}(X+\rho) / Q$,

which is less than the finite quantity $X^{\prime}$, and

$$
Q^{\prime \prime}=X^{\prime \prime}(X+\rho) / Q+\sigma^{2} X^{\prime 2} / Q^{3}
$$

which is similarly finite.

Let $\theta$ denote the greatest value of $i^{\prime \prime} Q^{\prime \prime} u^{2}+2 u \iota^{\prime}\left(2 Q^{\prime}-X^{\prime}\right)_{i}^{\prime} / u$ : taken us a fraction whose numerator and denominator are homogeneous quadratics in $u, u^{\prime}, u v^{\prime}$, the denominutor being essentially positive. By the ordinary 
methods we find that $Q \theta^{2}-Q^{\prime \prime} \theta-\left(2 Q^{\prime}-X^{\prime}\right)^{2}=0$. The roots of this quadratic are small quantities of the order of $Q^{-1}$, that is, of the order of $l^{-\frac{1}{b}}$.

Hence $u^{\prime \prime}=2 w(Q-X-\rho+\epsilon)$ where $\epsilon$ is a small quantity comparable with $7^{-\frac{1}{3}}$. Further,

$$
Q-l=|X+\lambda|-|\lambda|=\frac{2 \rho X+X^{2}}{|X+\lambda|+|\lambda|}
$$

Hence

$$
\therefore=\rho X / l+\mathrm{a} \text { small quantity comparable with } l^{-1} \text {. }
$$

Now

$$
Q-X-\rho=(l-\rho)(1-X / l)+\text { same error. }
$$

so that

$$
l-\rho=2 \beta^{2} \text {; }
$$$$
w "=\left(4 \beta^{2}+\eta\right) w,
$$

where $\eta$ is finite even when $l$ is made infinitely great. We shall suppose for the present that $\beta$ becomes infinite with $l$, which will happen if the amplitude of the complex quantity $\lambda$ is not too small.

Let $4 \mu_{1}^{2}$ and $4 \mu_{2}^{2}$ be the greatest and least values of $4 \beta^{2}+\eta$; then $\mu_{1}^{2}-\mu_{2}^{2}$ is always finite, and therefore $\mu_{1}-\mu_{2}, \mu_{1}-\beta, \beta-\mu_{2}$ tend to the limit zero as $\beta$ is increased, if $\mu_{1}, \mu_{2}$ have the same sign as $\beta$.

11. Now $y$ may stand for $\phi$ or $x$ : in the former case the values of $w, \imath v^{\prime}$ when $x=0$ are 1,0 ; so that, by the lemma (\$9), $v$ must lie hetween $\cosh 2 \mu_{1} x$ and $\cosh 2 \mu_{2} x$ and therefore $x$, or

$$
\left|(X+\lambda) \phi^{2}\right|+\left|\phi^{\prime}\right|^{2}=\frac{1}{2}\left(1+\epsilon_{1}\right) \exp 2 \beta x^{*}
$$

where $\epsilon_{1}$ tends to zero as $\beta$ is increased.

Similarly, $\quad\left|(X+\lambda) \chi^{2}\right|+\left|\chi^{\prime}\right|^{2}=\frac{1}{2}\left(1+\epsilon_{2}\right) \exp 2 \beta(1-x)$, where $\epsilon_{2}$ also tends to zero.

To find similar results for $\psi$ and $\omega$, let

Then

$$
w_{1}=\left|y^{2}\right|+\left|y^{\prime 2} /(X+\lambda)\right| \text {. }
$$

$$
\begin{aligned}
w_{1}^{\prime \prime}=2 u_{1}\left\{Q-X-\rho-Q^{\prime \prime} / 2 Q+Q^{\prime 2} / Q^{2} ;\right. & +u^{2}\left\{Q^{\prime \prime} / Q-2 Q^{\prime 2} / Q^{2}\right\} \\
& +4 \sigma Q^{\prime} u^{2} v^{\prime} / Q^{2}+2\left(Q^{2}-2 \sigma^{2}\right) X^{\prime \prime} u u^{\prime} / Q^{3} .
\end{aligned}
$$

Hence it comes out in the same way that

$$
\begin{aligned}
\left|\psi^{2}\right|+\left|\psi^{\prime 2} /(X+\lambda)\right| & =\frac{1}{2}\left(1+\epsilon_{3}\right) \exp 2 \beta x, \\
\left|\omega^{2}\right|+\left|\omega^{\prime 2} /(X+\lambda)\right| & =\frac{1}{2}\left(1+\epsilon_{4}\right) \exp 2 \beta(1-x),
\end{aligned}
$$

where $\epsilon_{9}, \epsilon_{1}$ tend to the limit zero as $\beta$ is increased.

- Here $\beta$ is taken positive: when $\beta$ is negative the value is $\frac{1}{2}\left(1+\epsilon_{1}\right) \exp (-2 \beta x)$, and so throughout what follows. 
12. Again, multiply the equation (1) by $2 y^{\prime}$ and integrate. Thus

Taling $\phi$ for $y$, we have

$$
y^{\prime 2}+(X+\lambda) y^{2}=\int X^{\prime} y^{2} d x+\text { const. }
$$

$$
\phi^{\prime 2}+(X+\lambda) \phi^{2}=1+\int_{0}^{x} X^{\prime} \phi^{2} d x,
$$

whence

$$
\left|\phi^{\prime 2}+(X+\lambda) \phi^{2}\right|<1+\xi \int_{0}^{x}\left\{\left|\phi^{\prime}\right|^{2}+\left|(X+\lambda) \phi^{2}\right|\right\} d x
$$

where $\dot{\xi}$ is the greatest numerical value of $X^{\prime} / Q$, and is therefore of the order of magnitude of $l^{-1}$.

Now $\left|\phi^{\prime}\right|^{2}+\left|(X+\lambda) \phi^{2}\right|$ increases with $x$, from 0 upwards, since it satisfies the equation $w^{\prime \prime}=\left(4 \beta^{2}+\eta\right) w$ and the initial conditions $w=1$, $w^{\prime}=0$. Hence in the integral in (8) the subject of integration is greatest at the upper limit, and

$$
\left|\phi^{\prime 2}+(X+\lambda) \phi^{2}\right|<1+\xi x\left\{\left|\phi^{\prime}\right|^{2}+\left|(X+\lambda) \phi^{2}\right|\right\} .
$$

Thus $\frac{\left|\phi^{2}+(X+\lambda) \phi^{2}\right|}{\left|\phi^{\prime}\right|^{2}+\left|(X+\lambda) \phi^{2}\right|}$ consists of two parts, one of the order of magnitude of $l^{-1}$ and the other of that of $\exp (-2 \beta x)$; both diminish indefinitely as $\beta$ increases, and therefore the ratio $\left|(X+\lambda) \phi^{2}\right| /\left|\phi^{\prime 2}\right|$ tends to the limit unity.

We may therefore say that $\left|\phi^{\prime}\right|^{2}$ and $\left|(X+\lambda) \phi^{2}\right|$, or in fact $\left|\lambda \phi^{2}\right|$, lie between the limits $\frac{1}{1}(1 \pm \epsilon) \exp 2 \beta x$, where $\epsilon$ diminishes indefinitely as $\beta$ increases. In the same way we have for $\left|\chi^{\prime}\right|^{2}$ and $\left|\lambda \chi^{2}\right|$ limits of the form $\frac{1}{4}(1 \pm \epsilon) \exp 2 \beta(1-x)$. Similarly, from the equation

$$
\frac{\psi^{\prime 2}}{X+\lambda}+\psi^{2}=1-\int_{0}^{x} \frac{X^{\prime}}{(X+\lambda)^{2}} \psi^{\prime 2} d x
$$

we deduce that $\left|\frac{\psi^{\prime 2}}{X+\lambda}+\psi^{2}\right|<1+\xi \int_{0}^{x}\left\{\left|\frac{\psi^{\prime 2}}{X+\lambda}\right|+\left|\psi^{2}\right|\right\} d x$

and that $\left|\psi^{2}\right|$ and $\left|\psi^{\prime 2} / \lambda\right|$ lie between limits of the form $\Varangle(1 \pm \epsilon) \exp 2 \beta x$; similarly the limits for $\left|\omega^{2}\right|$ and $\left|\omega^{2} / \lambda\right|$ are of the same form as those for $\left|\chi^{\prime}\right|^{2}$ and $\left|\lambda \chi^{2}\right|$.*

By putting $x=1$ in the results relating to $\phi$ and $\psi$ we find that $h^{2}, g^{2}, l e^{2}$, and $k^{2} / l$ lie between limits of the form $\frac{1}{4}(1 \pm \epsilon) \exp 2 \beta$.

13. The expression $\phi x \psi t-\phi t \psi x$ has also to be considered. As a function of $x$ it satisfies the equation (1) and the further conditions that when $x=t, y=0, y^{\prime}=1$. Hence, by analogy with the result found for $\phi$, 
$\left|\lambda(\phi x \psi t-\phi t \psi x)^{2}\right|$ lies between limits of the form $\frac{1}{2}(1 \pm \epsilon) \cosh 2 \beta(x-t)$, the hyperbolic function being used because the sign of $x-t$ is uncertain. $\left|\left(\phi^{\prime} x \psi t-\psi^{\prime} x \phi t\right)^{2}\right|$ also lies between limits of the same form.

Again, when $X=0, \psi=\phi^{\prime}$ and $g=h$. When $X$ is variable we have

$$
\phi^{\prime \prime \prime}+(X+\lambda) \phi^{\prime}+X^{\prime} \phi=0,
$$

and therefore

$$
\begin{aligned}
\left(\psi^{\prime \prime}-\phi^{\prime \prime \prime}\right)+(X+\lambda)\left(\psi-\phi^{\prime}\right) & =X^{\prime} \phi, \\
\left(\psi^{\prime \prime}-\phi^{\prime \prime \prime}\right) \phi-\left(\psi-\phi^{\prime}\right) \phi^{\prime \prime} & =X^{\prime} \phi^{2}, \\
\left(\psi^{\prime \prime}-\phi^{\prime \prime \prime}\right) \psi-\left(\psi-\phi^{\prime}\right) \psi^{\prime \prime} & =X^{\prime} \phi \psi .
\end{aligned}
$$

Thus

$$
\left(\psi^{\prime}-\phi^{\prime \prime}\right) \phi-\left(\psi-\phi^{\prime}\right) \phi^{\prime}=\int_{0}^{x} X^{\prime} \phi^{2} d x
$$$$
\left(\psi^{\prime}-\phi^{\prime \prime}\right) \psi-\left(\psi-\dot{\phi}^{\prime}\right) \psi^{\prime}=\int_{0}^{x} X^{\prime} \phi \psi d x,
$$

$$
\psi-\phi^{\prime}=\phi \int_{0}^{x} X^{\prime} \phi \psi d x-\psi \int_{0}^{x} X^{\prime} \phi^{2} d x=\int_{0}^{x} T^{\prime} \phi t(\phi x \psi t-\phi t \psi x) d t
$$

where $T^{\prime}$ is what $X^{\prime}$ becomes when $t$ is put for $x$.

But by our former work $\left|T^{\prime \prime} \phi t(\phi x \psi t-\phi t \psi x)\right|$, when $x>t$, does not exceed $C \exp \beta t \exp \beta(x-t) \div l$ where $C$ is a fixed finite quantity.

Hence $\psi-\phi^{\prime}$ does not exceed $C x \exp \beta x \div l$ : it therefore vanishes in the limit in comparison with $\psi$ or $\phi^{\prime}$, these being of the order of magnitude of $\exp \beta x$ : it follows that $g-h$ vanishes in comparison with $g$ or $h$.

14. Thus in the numerator of $\Omega(x, t, \lambda)$ the quantities $\phi x \chi^{t}, \psi x \times t$, $\phi x \omega t, \psi x \omega t, \phi x \psi t-\psi x \phi t$ are respectively of the same orders of magnitude ns $l^{-1} \exp \beta(1+x-t), \quad l^{-\frac{1}{3}} \exp \beta(1+x-t), \quad l^{-\frac{1}{1}} \exp \beta(1+x-t)$, $\exp \beta(1+x-t), l^{-\frac{3}{2}} \cosh \beta(x-t)$, while in the denominator $e, g, h, k$ are of the same orders as $l^{-\frac{1}{2}} \exp \beta, \exp \beta, \exp \beta, l^{\frac{1}{}} \exp \beta$. Thus when $x<t$ the terms of the numerator are small compared with those of the denominator, since $1+x-t$ and $t-x$ are both less than 1 : the numerator is therefore small in comparison with the denominator unless the terms of the latter neutralize each other. This cannot happen unless $K=0$, since $k$ is great compared with $e, g, h$. When $K=0$ the sum of the terms $G g+H h$ will only be of lower order than $g, h$ if $G=-H$, since we have proved that $g-h$ is small compared with $g$ or $h$ : this is, however, impossible, since, when $K=0, G H \Varangle L^{2}$, and therefore $G H$ cannot be negative. If then $K=0, G=-H$, we must have $G=H=L=0$, and only the terms containing $E$ are left.

Thus, when $x<t, \Omega(x, t, \lambda)$ tends to the limit zero as $\beta$ increases 
without limit: it is, in fact, comparable at most with the greater of the two* quantities $\quad l^{-\frac{1}{2}} \exp \beta(x-t), \quad l^{-\frac{1}{2}} \exp \beta(t-x-1)$.

The length of the contour of integration is comparable with $l$, and therefore $\int \Omega(x, t, \lambda) d \lambda$ tends to zero for those parts of the path where the amplitude of $\lambda$ is not nearly 0 or $2 \pi$, so that $\beta / \alpha$ is not small.

The part where the implitude of $\lambda$ is nearly 0 or $2 \pi$ must be treatel separately. When $X=0$, and the amplitude of $\lambda$ is $0, \phi$ and $\psi$ are simple trigonometrical functions. We need to know within what range the trigonometrical forms give a satisfactory approximation.

15. From the equation $\phi^{\prime \prime}+(X+\lambda) \phi=0$

we have $\quad \phi^{\prime} \sin x \sqrt{ } \lambda-\sqrt{ } \lambda \phi \cos x \sqrt{ } \lambda=-\int_{0}^{x} X \phi \sin x \sqrt{ } \lambda d x$ and $\quad \phi^{\prime} \cos x \sqrt{ } \lambda+\sqrt{ } \lambda \phi \sin x \sqrt{ } \lambda=1-\int_{0}^{x} X \phi \cos x \sqrt{ } \lambda d x$

by multiplying by $\sin x \sqrt{ } \lambda$ and $\cos x \sqrt{ } \lambda$ and integrating.

Eliminate $\phi^{\prime}$; then

$$
\sqrt{ } \lambda \phi=\sin x \sqrt{ } \lambda-\int_{0}^{r} T \phi(t) \sin (x-t) \sqrt{ } \lambda d t
$$

where $T$ stands for the same function of $t$ that $X$ is of $x$.

Suppose $\kappa$ to be a superior limit to $|\sqrt{ } \lambda \phi-\sin x \sqrt{ } \lambda|$. We have

$$
|\sin x \sqrt{ } \lambda| \ngtr \cosh \beta x
$$

where, as before,

$$
\lambda=(\alpha+\iota \beta)^{2} \text {. }
$$

Hence $|\phi \sqrt{ } \lambda| \ngtr \kappa+\cosh \beta x$ and $|\phi| \ngtr l^{-\frac{1}{1}}(\kappa+\cosh \beta)$, since $x \ngtr 1$.

Let $B$ be the greatest numerical value of $T$ between $t=0$ and $t=1$. Then

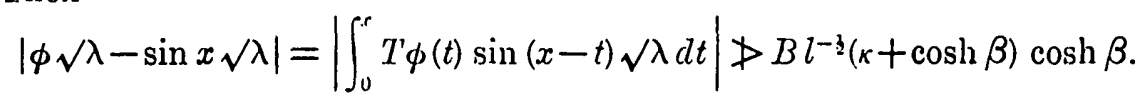

Thus

and we may take $\quad \kappa=B \cosh ^{2} \beta /\left(l^{\underline{k}}-B \cosh \beta\right)$.

This diminishes indefinitely as $l$ increases if $\cosh ^{2} \beta$ becomes infinitely small in comparison with $l^{\mathrm{l}}$, or with $\alpha$. This would happen if $\beta$ were $\ngtr \frac{1}{3} \log \alpha$. We have also

$$
\phi^{\prime}=\cos x \sqrt{ } \lambda-\int_{0}^{x} T \phi(t) \cos (t-x) \sqrt{ } \lambda d t,
$$

- The second must be taken when the term multiplied by $L$ is the most important in the numerator. Since $L$ does not vanish, $i, H, K$ cannot all vanish; so that the least favourable case is when $K^{-}=0$. When $h^{*}$ does not vanish $\Omega(x, t, \lambda)$ is comparable with $l^{-1} \exp \beta(x-t)$ or ulse: $l^{-1} \exp \beta(t-x-l)$. 
so that

$$
\left|\phi^{\prime}-\cos x \sqrt{ } \lambda\right| \ngtr B l^{-\frac{1}{b}}(\kappa+\cosh \beta) \cosh \beta,
$$

that is, $x$.

The same quantity $\kappa$ is a superior limit to $|\chi \sqrt{ } \lambda+\sin (1-x) \sqrt{ } \lambda|$ and $\left|x^{\prime}-\cos (1-x) \sqrt{ } \lambda\right|$, and also to $|\psi-\cos x \sqrt{ } \lambda|,\left|\psi^{\prime} \lambda^{-1}+\sin x \sqrt{ } \lambda\right|$, $|\omega-\cos (1-x) \sqrt{ } \lambda|,\left|\omega^{\prime} \lambda^{-k}-\sin (1-x) \sqrt{ } \lambda\right|$. Hence, by putting $X=0$ we get approximations to $\phi, \chi, \psi, \omega$ which hold good at least so long as $\beta$ is between $\pm \frac{1}{3} \log \alpha$. When $\beta$ does not lie between these limits the work of $\S \S 10-14$ is applicable, since $\beta$ increases indefinitely with $l$.

16. The path of integration may thus be divided into two parts, a minor arc on which $\alpha$ is great and positive and $\beta$ lies between $\pm \frac{1}{3} \log \alpha$, and a major arc on which $\beta$ lies without these limits. The value of the integral taken over the minor arc shows no sign of being infinitesimal, for the length of the arc becomes infinite and the subject of integration is of the order of magnitude of $l^{-\frac{1}{3}}$. Thus the series does not converge; in fact the successive terms of the series do not tend to zero as a limit.

If, however, we multiply the terms of the series by an analytical function of $t$, say, $F(t)$, and integrate with respect to $t$ between limits included in the range from 0 to 1 , the result is a convergent series whose sum to infinity can be found.

17. If $a, b$ are such that $0 \leqslant a<x<b \leqslant 1$, we have, choosing the expression for $\Sigma \Phi$ in which the contribution of the major arc is small,

$$
\begin{aligned}
2 \iota \pi \sum_{r=1}^{r=n} \int_{a}^{b} \Phi\left(x, t, \lambda_{r}\right) & F(t) d t \\
& =\iint_{a}^{x} \Omega(t, x, \lambda) F(t) d t d \lambda+\iint_{x}^{b} \Omega(x, t, \lambda) F(t) d t d \lambda,
\end{aligned}
$$

the integration with respect to $\lambda$ being again over the circle $|\lambda|=l$. which encloses $\lambda_{1}, \lambda_{2}, \ldots, \lambda_{n}$.

For $\Omega$ we may write $-(T+\lambda)^{-1} d^{2} \Omega / d t^{2}$ and integrate by parts with respect to $t$. The expression becomes

$$
\begin{aligned}
\iint_{a}^{x} \frac{d}{d t} \Omega(t, x, \lambda) & \frac{d}{d t} \frac{F(t)}{T+\lambda} d t d \lambda+\iint_{x}^{n} \frac{d}{d t} \Omega(x, t, \lambda) \frac{d}{d t} \frac{F(t)}{T+\lambda} d t d \lambda \\
& \quad \int\left[\sum_{t=a}^{t=x}\left\{\frac{d}{d t} \Omega(t, x, \lambda) \frac{F(t)}{T+\lambda}\right\}+\sum_{t=x}^{t=b}\left\{\frac{d}{d t} \Omega(x, t, \lambda) \frac{F(t)}{T+\lambda}\right\}\right] d \lambda .
\end{aligned}
$$

In the single integral pick out the terms given by substituting $x$ for $t$. 
They are

$$
\begin{aligned}
& -\int \frac{F^{\prime}(x)}{X+\lambda} ! \frac{E \phi^{\prime} \chi+G \psi^{\prime} \chi-H \psi^{\prime} \omega-K \psi^{\prime} \omega+L\left(\psi^{\prime} \psi^{\prime}-\phi w^{\prime} \prime^{\prime}\right)}{E c+G g+H h+K k-2 L} \\
& \left.-\frac{E \phi \chi^{\prime}+G \psi \chi^{\prime}-H \phi \omega^{\prime}-K \psi \omega^{\prime}+L\left(\phi \psi^{\prime}-\phi^{\prime} \psi\right)}{E e+G g+H h+K k-2 L}\right\} d \lambda .
\end{aligned}
$$

or $\int(X+\lambda)^{-1} F(x) d \lambda$ or $2 \iota \pi F(x)$, since the point where $\lambda=-X$ falls within the contour of integration. It is now to be proved that the rest of the expression tends to zero when the radius $l$ is increased indefinitely. The major and minor ares as defined above (\$16) must be considered separately.

18. Writing still $a+\imath \beta$ for $\sqrt{ } \lambda$, we have on the major arc a positive and $\beta>\frac{1}{3} \log \alpha$ numerically, and therefore increasing indefinitely with $l$. Then

$$
\begin{gathered}
\frac{d}{d t} \Omega(t, x, \lambda)=\left[E \phi^{\prime}(t) \chi(x)+G \psi^{\prime}(t) \chi(x)-H \phi^{\prime}(t) \omega(x)\right. \\
\left.-K \psi^{\prime}(t) \omega(x)+L \vdots \phi^{\prime}(t) \psi(x)-\psi^{\prime}(t) \phi(x)_{i}^{\prime}\right] \\
\div\left(E^{\prime} e+G g+H h+K k-2 L\right),
\end{gathered}
$$

which is at most* of the order of magnitude of $\exp \beta(x-t-1)$ or $\exp \beta(t-x)$, whichever is the higr er, and therefore tends to zero if $t<x$. Also $F(t)$ is finite and $d \lambda /(T+\lambda)$ nay be written

$$
[\lambda /(\lambda+T)](d \lambda / \lambda)
$$

in which expression the first factor is always finite, and the second is i $d \theta$ where $\lambda=l \exp \iota \theta$. The range of values of $\theta$ in the integration is less than $2 \pi$, and hence, for the major arc,

$$
\int\left\{\frac{d}{d l} \Omega(t, x, \lambda) \frac{F^{\prime}(t)}{T+\lambda}\right\}_{t=\imath} d \lambda
$$

tends to the limit zero.

Similarly

$$
\int\left\{\frac{d}{d t} \Omega(x, t, \lambda) \frac{F(t)}{T+\lambda}\right\}_{t=b} d \lambda,
$$

for the major arc, tends to zero, since $b>x$.

The first double integral may be written

$$
\text { ‘ } \iint_{a}^{x} \frac{d}{d t} \Omega(t, x, \lambda) \frac{d}{d t}\left\{\frac{\lambda}{\lambda+T} F(t)\right\} d t d \theta .
$$

8sR. 2. vOL. 3. No. 888 .

$$
\text { - See note to } \$ 14 .
$$

10. 888. 
The factor $\frac{d}{d t}\left\{\frac{\lambda}{\lambda+T} F(t)\right\}$ is always finite; let $A$ be a superior limit to its absolute value.

The factor $\frac{d}{d t} \Omega(t, x, \lambda)$, provided $a>x-\frac{1}{2}$, is not greater in absolute value than $B \exp \beta(t-x)$ where $B$ is also finite. Hence this double integral is not greater than

$$
2 \pi A B \int_{a}^{x} \exp \beta(t-x) d t \text { or } 2 \pi A B\{1-\exp \beta(a-x)\} / \beta,
$$

or than

$2 \pi A B \mid \beta$.

It therefore tends to the limit zero.

When $a<x-\frac{1}{2}$ the only difference is that for values of $t$ between $a$ and $x-\frac{1}{2}$ the term multiplied by $L$ becomes the most important in the numerator of $d \Omega / d t$, unless $L=0$. This does not affect the result: we have seen that, for such values of $t,|d \Omega / d t|$ has a superior limit of the form $B \exp \beta(x-t-1)$, and the integral of this between $a$ and $x-\frac{1}{2}$ is negligible.

The other double integral tends to zero similarly. Hence the whole contribution of the major are tends to the limit zero.

19. On the minor are the range of values of $\theta$ is infinitesimal, and hence it is only necessary to prove that the factors of the form $d \Omega / d t$ are finite in order to show that the contribution of the minor arc is infinitesimal. Clearly the arc must be so drawn as not to pass through any point such as $\lambda_{n}$.

Now on the minor arc $\beta<\frac{1}{3} \log \alpha$ numerically, and therefore the numerator and denominator of $\frac{d}{d t} \Omega(t, x, \lambda)$ differ by infinitesimals from $E \lambda^{-\frac{1}{2}} \cos t \sqrt{ } \lambda \sin (x-1) \sqrt{ } \lambda-G \sin t \sqrt{ } \lambda \sin (x-1) \sqrt{ } \lambda$

$-H \cos t \sqrt{ } \lambda \cos (x-1) \sqrt{ } \lambda+K \sqrt{ } \lambda \sin t \sqrt{ } \lambda \cos (x-1) \sqrt{ } \lambda+L \cos (x-t) \sqrt{ } \lambda$, and

$$
E \lambda^{-\frac{1}{2}} \sin \sqrt{ } \lambda+(G+H) \cos \sqrt{ } \lambda-K \sqrt{ } \lambda \sin \sqrt{ } \lambda-2 L .^{*}
$$

Suppose first that $K$ does not vanish; then the term containing it is the chief term in the denominator, the rest diminishing without limit in comparison, so long as $\sin \sqrt{ } \lambda$ is not small.

- This approximstion shows, if any proof is needed, that the series $\lambda_{1}, \lambda_{2}, \ldots$ is an infinite one, since by substituting successive even or odd multiples of $f \pi$ for $\sqrt{ } \lambda$ we find that the expression changes sign an infinite number of times. The even multiples of $\downarrow \pi$ serve when $K=0, G+H \neq 0$, the odd multiples in other cases. 
Now $|\sin \sqrt{ } \lambda|^{2}=\left|\sin ^{2}(\alpha+i \beta)\right|=\cosh ^{2} \beta-\cos ^{2} \alpha=\cosh ^{2} \beta$, if we take $\alpha=$ an odd multiple of $\frac{1}{2} \pi$. This implies that $\alpha$ is constant along the minor arc, which is therefore not circular, but parabolic; along it $\beta$ varies from $-\frac{1}{3} \log \alpha$ to $\frac{1}{3} \log \alpha$.

Similarly, $\quad\left|\sin ^{2} t \sqrt{ } \lambda\right|=\cosh ^{2} t \beta-\cos ^{2} t \alpha \ngtr \cosh ^{2} t \beta$, and

$$
\left|\cos ^{2} t \sqrt{ } \lambda\right|=\cosh ^{2} t \beta-\sin ^{2} t \alpha \ngtr \cosh ^{2} t \beta \text {. }
$$

Hence $|K \sqrt{ } \lambda \sin t \sqrt{ } \lambda \cos (x-1) \sqrt{ } \lambda| \ngtr K \sqrt{ } l \cosh t \beta \cosh (x-1) \beta$, which is less than $K \sqrt{ } l \cosh \beta$ when $t<x<1$; similarly

$$
|L \cos (x-t) \sqrt{ } \lambda| \ngtr L \cosh \beta,
$$

and so for the other terms of the numerator. Hence the contribution of the minor arc tends to zero when $K$ does not vanish.

Suppose next that $K=0$ : then $G H \varangle L^{2}$; so that $G+H$ cannot vanish unless $G, H, L$ are all zero. Take $G+H$ not to vanish. The same kind of proof applies, but, since

$$
|\cos \sqrt{ } \lambda|^{2}=\cosh ^{2} \beta-\sin ^{2} \alpha,
$$

we take $a$ to be a multiple of $\pi$; so that $|\cos \sqrt{ } \lambda|=\cosh \beta$.

If $G, H, K, L$ all vanish, the proof is the same as when $K$ is not zero.

Similarly for $\frac{d}{d t} \Omega(x, t, \lambda)$ wh on $x<t<1$.

20. Hence we have

when $a<x<b$.

$$
F(x)=\lim _{n=\infty} \sum_{r=1}^{r=n} \int_{a}^{b} \Phi\left(x, t, \lambda_{r}\right) F(t) d t
$$

If $x$ were $<a$, we should have

$$
2 \iota \pi \sum_{r=1}^{r=n} \int_{a}^{b} \Phi\left(x, t, \lambda_{r}\right) F(t) d t=\iint_{a}^{b} \Omega(x, t, \lambda) F(t) d t d \lambda,
$$

and this would be zero in the limit. Similarly, if $x>b$.

Thus $F(x)$ is expanded between $a$ and $b$ in a series of multiples of the functions $\phi\left(x, \lambda_{n}\right)$ and $\psi\left(x, \lambda_{n}\right)$; the sum of the series is zero when $0<x<a$ or $b<x<1$; the coefficients of $\phi\left(x, \lambda_{n}\right), \psi\left(x, \lambda_{n}\right)$ are the values when $\lambda=\lambda_{n}$ of

$$
\begin{aligned}
\int_{a}^{b}\{(E g+H k) \phi+(G g+K k-L) & \psi\} \\
& F(x) d x \\
\div & \int_{0}^{1}(E \phi \chi+G \psi \chi-H \phi \omega-K \psi \omega) d x
\end{aligned}
$$


and $\int_{a}^{b}\{(G g+K k-L) \phi-(G e+K h) \psi\} F(x) d x$

$$
\div \int_{0}^{1}(E \phi \chi+G \psi \chi-H \phi \omega-K \psi \omega) d x
$$

respectively; $\lambda_{1}, \lambda_{2}, \ldots$ are the values of $\lambda$ for which

$$
E e+G g+H h+K l:=2 L,
$$

and, if two of these coincide, the coefficients just written become indeterminate and are to be replaced by such quantities as are indicated by $(7), \S 8$.

It is easy to pass to a similar series which shall express different analytical functions in different parts of the range between 0 and 1 . The functions need not in fact be analytical, since only the first derivative of $F(x)$ was used in the proof; it is necessary to the proof that this derivative should exist, but the whole series of derivatives is not needed.

21. When $G H=E K+L^{2 *}$ the numerator of $\Phi(x, t, \lambda)$ breaks up into factors, one a function of $x$, and the other the same function of $t$. This happens in the best known special cases. For instance, when $G, H$, $K, L$ all vanish the equation determining $\lambda_{1}, \lambda_{2}, \ldots$ is $e=0$, and we have expansions in series of harmonic functions which all vanish at the two ends of the range of validity, since when $e=0$ the functions $\phi, \chi$ only differ by a constant factor.

Again, when $E=K=0, G=H=L=1$, each term of the expansion has the same value and the same derivative at the two ends of the range ; thus, if $X$ hall 1 as a period, the expansion would be in periodic functions; in our notation the type of such a function would be $x-\phi$ or $\omega-\psi$ indifferently.

22. Again, taking $X=0$, we have trigonometrical expansions, and, if ( $i=K=L=0$, the terms are of the form $A \sin a x$, the ralues of $a$ being such that

$$
\tan \alpha=-H \alpha / E \text {. }
$$

These are the expansions used by Fourier in his treatment of the problem

- Note that $G H-E K-L^{2}$ is an algebraic invariant of the two forms

$$
g h-e k-1, \quad E e+G g+H h+K k-2 L
$$

in the variables $e, g, h, k$. If this invariant is positive, and $e, g, h, k$ are taken as coordinates of a point in space of four dimensions, the two luci

$$
E e+G g+B h+K k-2 I=0, \quad g h-e k-1=0
$$

cut one another in a conicoid whose generaturs are imaginary, and, if the invariant vanishes, in a cone. 
of the conduction of heat in a solid sphere (see Burkhardt's Report, p. +20 : Fourier's Works, Vol. 1., p. 312). A variety of other forms of trigonometrical expansion could be derived by giving different values to $E, G, H, K, L$.

23. The question whether the expansion of $F(x)$ is valid when $x=a$ or $\forall$ has not yet been answered, but it is readily seen as in $\S 17$ that the expansion as a rule is only valid when $x=a$ if $F(a)=0$, and when $x=b$ if $F^{\prime}(b)=0$; more generally, the expansion of a discontinuous function does not hold good at the values where the discontinuities occur.

The cases $x=a=0$ and $x=b=1$ deserve special notice. Take first $x=a=0$. We have

$$
\begin{aligned}
2 \iota \pi & \sum_{r=1}^{n} \int_{0}^{h} \Phi\left(0, t, \lambda_{r}\right) F^{\prime}(t) d t \\
\quad & =\iint_{0}^{b} \frac{d}{d t} \Omega(0, t, \lambda) \frac{d}{d t} \frac{F(t)}{T+\lambda} d t d \lambda-\int_{t=0}^{t=h}\left\{\frac{d}{d t} \Omega(0, t, \lambda) \frac{F(t)}{T+\lambda}\right\} d \lambda .
\end{aligned}
$$

Now

$$
\frac{d}{d t} \Omega(0, t, \lambda)=\frac{G \chi^{\prime} t-k \omega^{\prime} t-L \phi^{\prime} t}{E e+G g+H h+K k-2 L},
$$

which is of the order of magnitude of $\exp (-\beta t)$, or possibly $\exp \beta(t-1)$ when $t>\frac{1}{2}$ and $L \neq 0$. Thu the double integral tends to zero as before. In the single integral th part given by substituting $b$ for $t$ tends to zero unless $b=1$; taking $b=1$ and writing $X_{0}, X_{1}$ for the values of $X$ when $x=0,1$, we have for the single integral

$\int\left\{\frac{G g+K k-L}{E e+G g+H h+K k-2 L} \frac{F(0)}{X_{0}+\lambda}-\frac{G-L h}{E e+G g+H h+K k-2 L} \frac{F(1)}{X_{1}+\lambda}\right\} d \lambda$.

The value of this is $2 \iota \pi F(0)$ if $X_{0}=X_{1}, E=0$, and

$$
H: L:: L: G:: F(1): F(0) \text {; }
$$

or if $F(1)=0=F(0)$; or if $E, G, H, L$ all vanish and $X_{0} \neq X_{1}$. It tends to the limit $2 \imath \pi F(0)$ if $F(0)=0$ and $K$ does not vanish, or if $F(0)=0$ and $K, L$ vanish.

Similarly, when $x=b=1$, we have

$$
\frac{d}{d t} \Omega(t, 1, \lambda)=\left(-H \psi^{\prime} t-K \psi^{\prime} t+L_{\chi^{\prime}} t\right) /(E e+G g+H h+K k-2 L),
$$

and the single integral in this case is, if $a=0$,

$$
\int\left\{(-H+L g) \frac{F(0)}{X_{0}+\lambda}-(-H h-K k+L) \frac{F(1)}{X_{1}+\lambda}\right\} \frac{d \lambda}{E e+G g+H h+K k-2 L} \text {. }
$$


The value of this is $2 \imath \pi F(1)$, if $X_{0}=X_{1}, E=0$, and

$$
H: L:: L: G:: F^{\prime}(1): F(0) \text {; }
$$

or if $F(1)=0=F(0)$; or if $E, G, H, L$ all vanish and $X_{0} \neq X_{1}$.

Hence the expansion is valid for the extreme values 0 and 1 in three crses, at least,

(1) When $F(1)=F(0)=0$.

(2) When $E, G, H, L$ vanish, so that the equation whose roots are $\lambda_{1}, \lambda_{2}, \ldots$ is $k=0$.

(3) When $X_{0}=X_{1}, E=0, L=m G, H=m^{2} G, F(1)=m F(0)$, $m$ being some resl constant, finite or infinite.

There are other cases in which the expansion is valid for one extreme value or the other.

It is easily seen that these conclusions are not affected by discontinuities in $F(x)$ at intermediate values of $x$.

24. It has been assumed that none of the critical points of the differential equation fall between the values 0 and 1 of the independent variable or at either of those values. If 0 or 1 were a critical point, the expansion wouid not necessariiy fail, as is shewn by the special case of Legendre's functions in which there is a critical point at each end of the range of validity. If the method of this paper were used, the initial conditions by which $\phi, \chi, \psi, \omega$ are defined would have to be modified.

\section{NOTES ADDED IN PROOF.}

I. To the results in $\$ \$ 6,7$ the following may be added. If $Y$ stands for $E e+G g+H h+K k-2 L, Y=0$ is an equation for $\lambda$ whose roots are separated by those of $E g+H k=0$, also by those of $G e+K h=0$, and further by those of $Z+\kappa Y=0$, where

$$
\begin{aligned}
Z=a^{2}(E g+H k)+\beta^{2}(E h+G k)-\gamma^{2}(H e+K g) & -\delta^{2}(G e+K h) \\
& -2(a \delta+\beta \gamma) M,
\end{aligned}
$$

$a, \beta, \gamma, \delta, \kappa$ being any real constants, and $M$ a real constant such that

$$
M^{2} \ngtr G H-E K-L^{2} .
$$


It follows, in fact, from the formule of the paper that

$$
\begin{aligned}
Z \dot{Y}-Y \dot{Z}=\int_{0}^{1}\left[\left\{\alpha\left(E_{\chi}-H \omega+L \psi\right)-\delta M \phi\right\}^{2}+\{\beta(E \phi+G \psi-L \omega)-\gamma M \chi\}^{2}\right. \\
+\{\gamma(H \phi+K \psi-L \chi)+\beta M \omega\}^{2}+\{\delta(G \chi-K \omega-L \phi)-\alpha M \psi\}^{2} \\
\\
\left.+\left(\alpha^{2} \psi^{2}+\beta^{2} \omega^{2}+\gamma^{2} \chi^{2}+\delta^{2} \phi^{2}\right)\left(G H-E K-L^{2}-M^{2}\right)\right] d x .
\end{aligned}
$$

'This being never negative, the roots of $Y=0$ and $Z+{ }_{\kappa} Y=0$ separate each other as a rule; it is, however, possible for $Y, Z$ to vanish together when $G H-E K=L^{2}+M^{2}$. If $\lambda, \lambda_{1}$ were conjugate complex quantities, we should have $\left(Z Y_{1}-Y Z_{1}\right) /\left(\lambda_{1}-\lambda\right)$ essentially positive.

II. 'The results of $\$ \$ 11-13$ may be proved, perhaps more simply, by the following method. Suppose, in $\$ 15$, that $\beta$ is not restricted, and that $\kappa$ is a superior limit to

$$
|\sqrt{ } \lambda \phi-\sin x \sqrt{ } \lambda| / \cosh \beta x \text {. }
$$

'I'hen

$$
|\phi \sqrt{ } \lambda| \ngtr(1+\kappa) \cosh \beta x ;
$$

thus $\quad|\phi \sqrt{ } \lambda-\sin x \sqrt{ } \lambda| \ngtr B l^{-1}(1+\kappa) \int_{0}^{x} \cosh \beta t \cosh \beta(x-t) d t$

$$
\ngtr B l^{-\frac{b}{3}}(1+\kappa)\left(\frac{x}{2} \cosh \beta x+\frac{1}{2 \beta} \sinh \beta x\right) ;
$$

so that $\kappa \ngtr B l^{-k}(1+\kappa)$, since ta. h $\beta x<\beta x, x<1$.

Hence we may take $\kappa=B /(:-B)$, a small quantity, and the ratio of $\phi \sqrt{ } \lambda$ to $\sin x \sqrt{ } \lambda$ tends to unity when $\beta$ is increased indefinitely, since $|\sin x \sqrt{ } \lambda|$ approaches equality with $\cosh \beta x$, and $\phi \sqrt{ } \lambda-\sin x \sqrt{ } \lambda$ is small compared with $\cosh \beta x$. In the same way $\phi^{\prime}$ and $\cos x \sqrt{ } \lambda, \psi$ and $\cos x \sqrt{ } \lambda$, $\psi^{\prime}$ and $-\sqrt{ } \lambda \sin x \sqrt{ } \lambda, \chi \sqrt{ } \lambda$ and $\sin (x-1) \sqrt{ } \lambda, \chi^{\prime}$ and $\cos (x-1) \sqrt{ } \lambda$, $\omega$ and $\cos (x-1) \sqrt{ } \lambda, \omega^{\prime}$ and $\sqrt{ } \lambda \sin (1-x) \sqrt{ } \lambda$ tend respectively to equality when $\beta$ is increased indefinitely. 